\title{
De Novo Endometrial Implant Into the Colon After Uterine Morcellation
}

\author{
Erin M. Mellano, MD, Christy M. Anthony, BS, Tamara Grisales, MD, Christopher M. Tarnay, MD \\ Department of Obstetrics and Gynecology, Division of Female Pelvic Medicine and Reconstructive Surgery, University of \\ California, Los Angeles, David Geffen School of Medicine, Los Angeles, CA, USA (all authors).
}

\begin{abstract}
Instances of iatrogenic endometriosis after laparoscopic supracervical hysterectomy are uncommon but have been reported in the literature in women undergoing hysterectomy for pelvic pain. A 41-year-old woman with no history of endometriosis developed cyclic pain and a mass in her lower abdominal wall after uterine morcellation for a laparoscopic supracervical hysterectomy and sacrocolpopexy for uterocervical prolapse. Exploratory surgery revealed an endometrial implant involving the peritoneum and omentum, with transmural involvement of the cecum at a site separate from the trocar site. Aberrant endometrial cell implantation after morcellation of the uterine corpus has been reported; however, this was a rare instance of transmural large bowel iatrogenic endometriosis, necessitating a partial bowel resection, in a patient without a history of endometriosis or pelvic pain, at a site remote from where the uterus was morcellated and removed. In light of recent concerns over dissemination of occult cancerous cells, this case illustrates that dissemination of noncancerous cells can similarly have detrimental outcomes for patients.
\end{abstract}

Key Words: Endometriosis, Laparoscopy, Supracervical hysterectomy, Uterine morcellation.

Citation Mellano EM, Anthony CM, Grisales T, Tarnay CM. De novo endometrial implant into the colon after uterine morcellation. CRSLS e2014.00403. DOI 10.4293/CRSLS.2014.00403.

Copyright (C) 2014 SLS This is an open-access article distributed under the terms of the Creative Commons Attribution-Noncommercial-ShareAlike 3.0 Unported license, which permits unrestricted noncommercial use, distribution, and reproduction in any medium, provided the original author and source are credited.

Address correspondence to: Erin M. Mellano, MD, University of California, Los Angeles, David Geffen School of Medicine, Department of Obstetrics and Gynecology, Division of Female Pelvic Medicine and Reconstructive Surgery, 10833 Le Conte Avenie, Box 951740, Los Angeles, CA 90095-1740, USA. Telephone: 310-267-5918, Fax: 310-794-6635; E-mail: emellano@mednet.ucla.edu

\section{INTRODUCTION}

Endometriosis is defined by the presence of endometrial glands and stroma outside the lining of the endometrial cavity. Sites that may be seeded with these endometrial glands include the ovaries, peritoneum, rectovaginal septum, abdominal wall, bowel, ureter, and even lung parenchyma. ${ }^{1,2}$ Endometriosis affects approximately $6 \%$ to $10 \%$ of all reproductive-aged women and accounts for $35 \%$ to $50 \%$ of women who experience cyclic chronic pelvic pain and/or have complications with fertility. The severity of the symptoms differs greatly among these women and has no correlation with the extent of disease. ${ }^{2}$

Cases of postoperative iatrogenic endometriosis after pelvic surgery are rare. In women with known histories of endometriosis, surgical scar endometriomas have been documented after surgeries that involve the uterus, such as a Cesarean section or hysterectomy. ${ }^{3-5}$ The proposed mechanism for trocar-site or surgical-scar endometriosis is spillage of endometrial cells at the time of surgery, im- plantation of these cells, and proliferation to form endometriomas. ${ }^{6}$

More than 200,000 women undergo surgical correction for pelvic organ prolapse each year. Advances in minimally invasive surgical techniques facilitate the ability of surgeons to perform sacrocolpopexy procedures for vault prolapse with decreased morbidity.7,8 For women who have uteri at the time of the procedure, a supracervical hysterectomy is generally performed to allow a durable repair, while minimizing the risk for mesh complications. As the number of laparoscopic sacrocolpopexy procedures increases, uterine morcellation will be performed more frequently.

Uterine morcellation may potentiate dissemination of endometrial cells in the peritoneal cavity. In reproductiveaged women, abnormal seeding of endometrial cells may respond to hormonal fluxes and produce new-onset symptoms of endometriosis in women with histories of it. Evidence of implantation of endometriomas after laparo- 
scopic supracervical hysterectomy has been documented in women without evidence of the disease before surgery. In these cases, the patients undergoing hysterectomy preoperatively reported dysmenorrhea or pelvic pain suggesting preexisting endometriosis. ${ }^{9-13}$ The present case report is unique in that an endometrial implant developed well away from the site of morcellation, involving the large bowel and peritoneum, in a woman with no history of pelvic pain or signs of endometriosis at the time of her index operation.

\section{CASE}

A 41-year-old, parous 2 woman was referred to our office for symptomatic stage 3 pelvic organ prolapse and urinary stress incontinence for which she desired surgical correction. She underwent a laparoscopic supracervical hysterectomy, sacrocolpopexy, posterior colporrhaphy, perineorrhaphy, retropubic midurethral sling, and cystoscopy. The procedure was uncomplicated, and the pelvic anatomy was visually normal, including the ovaries, which were left in situ. The uterus was morcellated intraperitoneally with a power morcellator and was removed through a $12-\mathrm{mm}$ umbilical incision under direct visualization with the camera in the $12-\mathrm{mm}$ right lower quadrant assistant port. Her postoperative course was uncomplicated, and she was discharged home on postoperative day 2 .

Approximately 5 months after her surgery, the patient began to experience cyclic pain and vaginal spotting. The vaginal spotting was attributed to residual endometrial glands in the endocervical canal. She had no history of dysmenorrhea and no overt endometriosis noted at the time of her surgery. The results of physical examination were unremarkable, and an in-office ultrasound study revealed normal-appearing adnexal structures. She was treated with nonsteroidal anti-inflammatory medication for her pain.

Over the next year, she continued to be vexed with right low abdominal wall pain at the site of a self-reported abdominal firmness that expanded and regressed in size cyclically with menstruation. Interval imaging studies, including ultrasound and computed tomography (CT), were performed and were unable to locate an abnormality in the anterior abdominal wall. Of note, an inflammatory focus along the lower margin of the cecum in the right lower quadrant, $15 \mathrm{~mm}$ in size with some fat stranding and trace fluid, interpreted as postsurgical changes, was seen on CT $>12$ months after her surgery. Attempts to treat her pain with local anesthetic and oral analgesics were largely

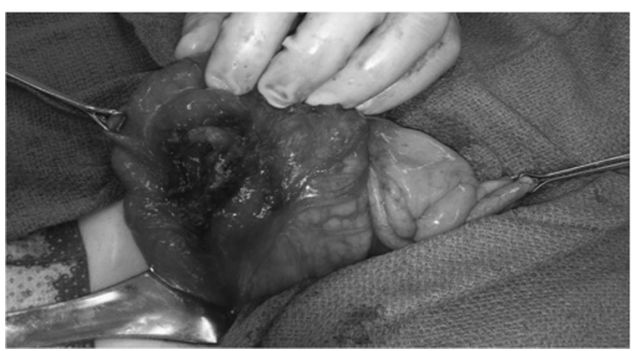

Figure 1. Cecal involvement of endometriotic implant.

unsuccessful. Given the cyclic nature of the pain, a 1-month trial of a gonadotropin-releasing hormone agonist was administered. The patient noticed a dramatic improvement of her pain with hormonal suppression.

Eighteen months after surgery, she was examined while on her menstrual cycle, and a palpable mass was appreciated, medial and inferior to the right lower quadrant assistant laparoscopic port site in the anterior abdominal wall. Her symptoms and the response to hormonal suppression were suggestive of endometriosis, despite uncharacteristic location, distant from the umbilical port where the morcellation and uterine tissue had been removed. Magnetic resonance imaging (MRI) performed during menstruation did not demonstrate any visually enhancing lesions in the abdominal wall. Despite nondiagnostic imaging results, the presumptive clinical conclusion was an endometriotic implant. Given the debilitating nature of this pain, the patient elected to undergo an exploration and excision of this mass.

On examination under anesthesia, a $3 \times 4 \mathrm{~cm}$ palpable mass in the anterior abdominal wall was identified. The palpable mass was below the level of the fascia, and upon entry into the peritoneum, a lobulated $3 \times 5 \mathrm{~cm}$ mass was encountered. This was adherent to the peritoneum and omentum, with a gross appearance consistent with endometriosis. The conglomerate involved the nearby viscera, with transmural involvement of the cecum (Figure 1). The mass, a portion of the cecum, and the adjacent appendix were resected with the assistance of a general surgeon (Figure 2).

Intraoperative frozen section was consistent with endometriosis. No additional lesions or masses were visualized in the peritoneal cavity. The patient had an uneventful recovery and was discharged home 2 days postoperatively. Final pathology confirmed the mass to be an irregular $5 \times 3.4 \times 2.0 \mathrm{~cm}$ fragment of yellow-gray, fibrofatty, indurated tissue with focal cavities filled with blood, sug- 


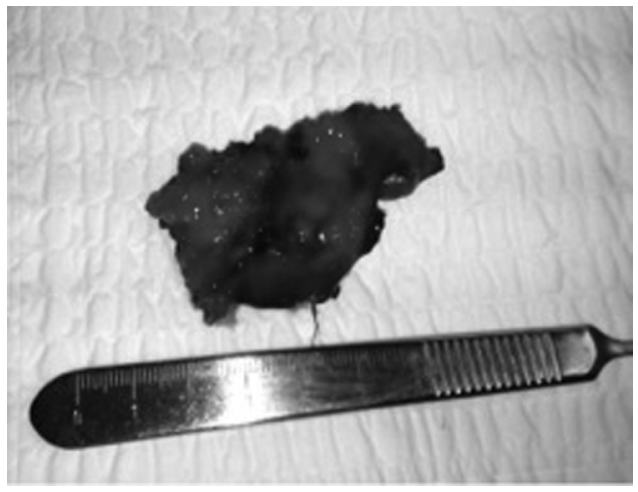

Figure 2. Gross specimen of endometrial implant sent to pathology.

gestive of endometrioma. The cecum was noted to have transmural infiltration of the endometrioma.

\section{DISCUSSION}

Our patient presented postoperatively with a clinical picture classic for endometriosis, with cyclic pain and a palpable, tender mass on examination. Although cases of iatrogenic endometriotic implants have been reported after pelvic surgery, this case is unique in that the patient had no history suggestive of endometriosis, nor were there any intraoperative findings of endometriosis at the time of her index surgery. Case reports of de novo endometriosis after uterine morcellation have been notable in patients with preoperative symptoms of pelvic pain, suggestive of preexisting endometriosis. ${ }^{9-13}$

The absence of preoperative pelvic pain or intraoperative signs of preexisting endometriosis implies that the most likely etiology of our patient's endometrioma was direct seeding of endometrial cells after morcellation. What is interesting about this particular case is that the endometriotic implant did not involve a laparoscopic port site. Furthermore, it was just inferior to the right lateral trocar site, where the laparoscope was used during morcellation through the umbilical port. It is possible that the viewing angle in relationship to the right lower quadrant port site precluded the surgeons from recognizing a fragment of retained uterine tissue.

Traditional imagining techniques used to diagnose endometriosis include ultrasound, MRI, and CT. It is unusual to be unable to identify abdominal wall endometriotic implants with MRI. The sensitivity of MRI in detecting endometriosis ranges from $78 \%$ to $96.3 \%$, with specificity of $73 \%$ to $100 \%{ }^{14-16}$ Although the palpable lesion in this patient was thought to be in the anterior abdominal wall, the true transcecal location made it significantly more challenging to identify on imaging. Ultrasound, doublecontrast barium enema, CT, and MRI have all been used to diagnose bowel endometriosis, but they have limitations. ${ }^{17}$ The sensitivity of these techniques can be reduced by bowel peristalsis, even after bowel preparation. An antispasmodic may be helpful in visualizing intestinal endometriosis by preventing artifacts from peristalsis. ${ }^{15} \mathrm{Gad}-$ olinium may help enhance a study, but there is no consensus of benefit. Recent studies advocate for the use of 3.0-T MRI to increase sensitivity and specificity in detecting deep infiltrating endometriosis, compared with the routine 1.5-T pelvic MRI protocol. ${ }^{14}$ Regardless of imaging modality, high clinical suspicion should not be dissuaded by negative imaging.

A laparoscopic supracervical hysterectomy performed at the time of colpopexy mandates that the uterus be morcellated to remove the specimen. With advances in minimally invasive endoscopic surgery, surgeons should be aware of the potential complications, although the overall incidence of these complications is difficult to determine. Clearly, morcellating devices have the potential for great visceral injury, but the risks associated with dissemination of small tissue fragments are more difficult to delineate. Case repots of ectopic leiomyoma, endometriosis, adenomyosis, ovarian tissue, and sarcoma exist in the literature, but the overall incidence is unknown. ${ }^{18}$ In a case-control study comparing the rate of de novo endometriosis after hysterectomy with and without morcellation, the overall incidence was found to be $1 \%$ in both groups, suggesting no additional risk with morcellation. ${ }^{6}$ Instances of atypical endometrial implants found years after supracervical hysterectomy with benign pathology demonstrate the potential capability of transformation into abnormal histology. ${ }^{2}$ Even more rare are cases of leiomyomatosis peritonealis disseminata after morcellation of leiomyoma for benign indications, quoted at a rate of $0.1 \% .{ }^{19}$

The risk for disseminating occult cancerous cells with morcellation is low, but the morbidity of such an occurrence is significant. The baseline rate of port-site metastases in laparoscopic gynecologic oncology surgery is $1 \%$ to $2 \%$, which is similar to the incisional metastatic rate for equivalent exploratory laparotomy procedures. ${ }^{20}$ The American College of Obstetricians and Gynecologists recommends that adequate assessment of the endometrial cavity be performed before morcellation; however, this is not routinely done for asymptomatic women who are undergoing surgery for prolapse. ${ }^{21,22}$ Endometrial biopsy is a sensitive and specific method for identifying endometrial abnormalities, but there are no reliable diagnostic 
tests to identify rare instances of leiomyosarcoma (LMS). ${ }^{18,23}$ LMS is typically diagnosed after surgery for a presumed leiomyoma. The incidence of unexpectedly diagnosing a leiomyoma variant, leiomyoma atypia, or a malignant sarcoma after morcellation is estimated at $0.09 \%$ to $1 \% .{ }^{18,19,24}$ The Society of Gynecologic Oncology released a statement in December 2013 that morcellation is contraindicated in the presence of documented or suspected malignancy and may be inadvisable in premalignant conditions or risk-reducing surgery. ${ }^{25}$ Morcellation of LMS increases the rate of abdominopelvic dissemination and adversely affects disease-free and overall survival. ${ }^{23}$ In cases of occult sarcoma after morcellation, evidence of peritoneal dissemination was seen up to $64 \%$ of the time on subsequent evaluation. ${ }^{19,24}$ Regardless of whether the tumor is removed intact or morcellated, the Society of Gynecologic Oncology statement emphasizes that LMS has an extremely poor prognosis. 25

Current power morcellation devices do not provide a fail-safe method for preventing intraperitoneal spread of morcellated tissue. Ultimately, the only way to avoid the spread of cells of the morcellated tissue would be to have an enclosed morcellation system. Some have suggested that specimens should be placed in endoscopic bags and brought up to the port site to be manually morcellated for removal. ${ }^{18}$ This is a cumbersome technique that may not be feasible for large or partially calcified specimens. Increased interest in developing devices to prevent spill during morcellation have been reported, such as power morcellation within a bag or special enclosed wire-mesh morcellators. ${ }^{18,26}$ As of yet, these devices are experimental, and until contained morcellation devices become available, precautions should be taken to prevent peritoneal spread or seeding of morcellated tissues. We suggest irrigation of the peritoneal cavity, along with careful inspection through an additional port site to ensure that no fragments of tissue are missed. In this particular case, reevaluating the peritoneal cavity from the umbilical central port site might have identified retained tissue previously unrecognized. As we grapple with the ramifications of morcellation in the rare cases of cancer, this case highlights the sequelae of morcellation even in benign disease. In concordance with the recommendations of the Society of Gynecologic Oncology and the American College of Obstetricians and Gynecologists, surgeons should have transparent discussions with their patients regarding risks, benefits, and alternatives of all procedures so that patients can make informed decisions in their medical care. ${ }^{22,25}$

\section{References:}

1. Horton JD, Dezee KJ, Ahnfeldt EP, Wagner M. Abdominal wall endometriosis: a surgeon's perspective and review of 445 cases. Am J Surg. 2008;196(2):207-212.

2. Burney RO, Giudice LC. Pathogenesis and pathophysiology of endometriosis. Fertil Steril. 2012;98(3):511-519.

3. Dwivedi AJ, Agrawal SN, Silva YJ. Abdominal wall endometriomas. Dig Dis Sci. 2002;47(2):456-461.

4. Song JY, Borncamp E, Mehaffey P, Rotman C. Large abdominal wall endometrioma following laparoscopic hysterectomy. JSLS. 2011;15(2):261-263.

5. Leite GK, Carvalho LF, Korkes H, et al. Scar endometrioma following obstetric surgical incisions: retrospective study on 33 cases and review of the literature. Sao Paolo Med J. 2009;127(5): $270-277$.

6. Schuster MW, Wheeler TL II, Richter HE. Endometriosis after laparoscopic supracervical hysterectomy with uterine morcellation: a case control study. J Minim Invasive Gynecol. 2012;19(2): 183-187.

7. Paraiso MR, Walters MD, Rackley RR, Melek S, Hugney C. Laparoscopic and abdominal sacral colpopexies: a comparative cohort study. Am J Obstet Gynecol. 2005;192(5):1752-1758.

8. Klauschie JL, Suozzi BA, O'Brien MM, McBride AW. A comparison of laparoscopic and abdominal sacral colpopexy: objective outcome and perioperative differences. Int Urogynecol J Pelvic Floor Dysfunct. 2009;20:273-279.

9. Kill LM, Kapetanakis V, McCullough AE, Magrina JF. Progression of pelvic implants to complex atypical endometrial hyperplasia alter uterine morcellation. Obstet Gynecol. 2011; 117(2 Pt 2):447-449.

10. Sepilian V, Della Badia C. Iatrogenic endometriosis caused by uterine morcellation during a supracervical hysterectomy. Obstet Gynecol. 2003;102(5 Pt 2):1125-1127.

11. Wilson H, Shaxted E. Implantation endometrioma at port site after laparoscopic abdominal supracervical hysterectomy. Gynaecol Endosc. 1999;8:245-247.

12. Bektaş H, Bilsel Y, Sari YS, et al. Abdominal wall endometrioma; a 10-year experience and brief review of the literature. J Surg Res. 2010;164(1):e77-e81.

13. Seydel AS, Sickel JZ, Warner ED, Sax HC. Extrapelvic endometriosis: diagnosis and treatment. Am J Surg. 1996;171(2):239.

14. Bianek-Bodzak A, Szurowska E, Sawicki S, Liro M. The importance and perspective of magnetic resonance imaging in the evaluation of endometriosis. Biomed Res Int. 2013;2013: 436589 .

15. Chamié LP, Blasbalg R, Gonçalves, Carvalho FM, Abrão MS, de Oliveira IS. Accuracy of magnetic resonance imaging 
for diagnosis and preoperative assessment of deeply infiltrating endometriosis. Int J Gynaecol Obstet. 2005;90: $218-222$.

16. Bazot M, Lafont C, Rouzier R, Roseau G, Thomassin-Naggara I, Daraï E. Diagnostic accuracy of physical examination, transvaginal sonography, rectal endoscopic sonography and magnetic resonance imaging to diagnose deep infiltrating endometriosis. Fertil Steril. 2009;92(6):1825-1833.

17. Choudhary S, Fasih N, Papadatos D, Venkateswar SR. Unusual imaging appearances of endometriosis. AJR Am J Roentgenol. 2009;192:1632-1644.

18. Kho KA, Nezhat $\mathrm{CH}$. Evaluating the risks of electric uterine morcellation. JAMA. In press.

19. Sizzi O, Rossetti A, Malzoni M, et al. Italian multicenter study on complications of laparoscopic myomectomy. J Minim Invasive Gynecol. 2007;14(4):453-462.

20. Ramirez PT, Wolf JK, Levenback C. Laparoscopic port-site metastases: etiology and prevention. Gynecol Oncol. 2003;91(1): 179-189.

21. Ramm O, Gleason JL, Segal S, Antosh DD, Kenton KS. Utility of preoperative endometrial assessment in asymptomatic women undergoing hysterectomy for pelvic floor dysfunction. Int Urogynecol J. 2012;23:913-917.

22. American College of Obstetricians and Gynecologists. ACOG Committee Opinion 388: supracervical hysterectomy. November 2007, reaffirmed 2013. Available at: http://www.acog.org/Resources_And_ Publications/Committee_Opinions/Committee_on_Gynecologic_ Practice/Supracervical_Hysterectomy. Accessed February 11, 2014.

23. Park JY, Park SK, Kim DY, et al. The impact of tumor morcellation during surgery on the prognosis of patients with apparently early uterine leiomyosarcoma. Gynecol Oncol. 2011; 122(2):255-259.

24. Seidman MA, Oduyebo T, Mutu MG, Crum CP, Nucci MR, Quade BJ. Peritoneal dissemination complicating morcellation of uterine mesenchymal neoplasms. PLoS One. 2012;7(11):e50058.

25. Society of Gynecologic Oncology. Morcellation. December 2013. Available at: https://www.sgo.org/newsroom/positionstatements-2/morcellation/. Accessed February 12, 2014.

26. Isakov A, Murdaugh KM, Burke WC. A new laparoscopic morcellator using an actuated wire mesh and bag. J Med Devices. 2014;8(1):110091-110097. 